\title{
The analysis of differential diagnosis of benign and malignant thyroid nodules based on ultrasound reports
}

\author{
Shumei Miao ${ }^{1,2}$, Mang Jing ${ }^{1,2}$, Rongrong Sheng ${ }^{1,2}$, Dai Cui ${ }^{3}$, Shan Lu $^{4}$, Xin Zhang ${ }^{1,2}$, Shenqi Jing ${ }^{1,2}$, \\ Xiaoliang Zhang ${ }^{1,2}$, Tao Shan ${ }^{1,2}$, Hongwei Shan ${ }^{1,2}$, Tingyu $\mathrm{Xu}^{1}$, Bing Wang ${ }^{1}$, Zhongmin Wang ${ }^{2}$, \\ Yun $\mathrm{Liu}^{2}$ \\ ${ }^{1}$ Department of Information, The First Affiliated Hospital of Nanjing Medical University, Nanjing 210096, China; ${ }^{2}$ Department of Medical \\ Informatics, School of Biomedical Engineering and Informatics, Nanjing Medical University, Nanjing 211166, China; ${ }^{3}$ Department of \\ Endocrinology, ${ }^{4}$ Health Education Section, The First Affiliated Hospital of Nanjing Medical University, Nanjing 210096, China \\ Contributions: (I) Conception and design: S Miao, Z Wang, Y Liu, D Cui, S Lu, X Zhang; (II) Administrative support: Y Liu, Z Wang; (III) Provision \\ of study materials or patients: S Miao, M Jing, S Jing, H Shan; (IV) Collection and assembly of data: S Miao, R Sheng, X Zhang; (V) Data analysis \\ and interpretation: S Miao, R Sheng, T Shan, T Xu; (VI) Manuscript writing: All authors; (VII) Final approval of manuscript: All authors. \\ Correspondence to: Yun Liu, PhD; Zhongmin Wang, PhD. Department of Medical Informatics, School of Biomedical Engineering and Informatics, \\ Nanjing Medical University, Nanjing 211166, China. Email: liuyun@njmu.edu.cn; wzm.cn@139.com.
}

Background: Thyroid cancer is a common endocrine tumor, the incidence of which is increasing each year. Early diagnosis and treatment can effectively prevent thyroid cancer. This article uses Chinese's ultrasound reports to determine the value of early diagnosis.

Methods: The clinical data center of the First Affiliated Hospital of Nanjing Medical University was screened for patients diagnosed with a thyroid nodule, who had undergone a thyroid function test, ultrasound records and pathological assessment. A total of 811 patients with a total of 1,290 pathologically confirmed nodules (506 benign and 784 malignant) were enrolled. Logistic regression was used to analyze the variables that significantly affected malignant nodules. The sensitivity and specificity of ultrasound thyroid imagingreporting and data system (TI-RADS) classification results for benign and malignant tumors were calculated. Results: The age of the patients had a very significant difference in the classification of benign and malignant nodules $(\mathrm{P}<0.001)$, and the marital status was significantly different $(\mathrm{P}<0.05)$. Gender and medical insurance had no significant effect $(\mathrm{P}>0.05)$. Thyroglobulin (TG), free thyroxine (FT4), and free triiodothyronine (FT3) had significant effects $(\mathrm{P}=0.003)$ on the incidence of malignant nodules in patients, while thyroid-stimulating hormone (TSH) had no significant effect $(\mathrm{P}>0.05)$. Ultrasound analysis showed a Youden's index of $78.97 \%$, a positive predictive value of $93.20 \%$, and a negative predicted value of $84.10 \%$ at the most excellent classification effect. The sensitivity was $89.0 \%$, the specificity was $89.9 \%$; much greater than the classification model based on the thyroid function test (sensitivity $=80.6 \%$, specificity $=55.8 \%$ ).

Conclusions: The present study verifies the effectiveness of using TI-RADS classification for diagnosis of benign and malignant thyroid nodules, and explores the use of new analysis methods for clinical data. To reduce dependence on the doctors, ultrasound image data and clinical phenotypic data can be further used to assist clinical decision making.

Keywordsa Pathological; thyroid; thyroid imaging-reporting and data system (TI-RADS); ultrasound

Submitted Dec 12, 2019. Accepted for publication Mar 12, 2020.

doi: $10.21037 /$ gs.2020.04.03

View this article at: http://dx.doi.org/10.21037/gs.2020.04.03 


\section{Introduction}

Thyroid cancer is one of the most common types of cancer in the endocrine system and accounts for more than $10 \%$ of malignant tumors (1), which is much higher than the incidence of other head and neck tumors. In recent years, the incidence of thyroid cancer has increased year by year; now it is the fifth most common cancer diagnosed in females, and it is expected to become the second most common malignancy in females and the ninth most common malignancy in males by the year 2030 (2). The study of thyroid nodules has received extensive attention from clinicians and researchers. Some thyroid nodules have a high cancer rate. Early diagnosis and treatment of thyroid nodules can effectively prevent thyroid cancer and avoid serious consequences $(3,4)$. How benign and malignant nodules should be identified from patients' clinical data, and how malignant nodules should be identified from a large number of thyroid nodules are very important and challenging considerations in the clinic (5).

In clinical practice, neck ultrasound and fine-needle aspiration (FNA) of thyroid nodules are often used for primary diagnosis. Ultrasound is noninvasive, convenient, and inexpensive, and it does not expose the patient to damaging radiation. Thus, ultrasound is preferred for screening thyroid nodules and for carrying out preoperative assessment compared with the more expensive and invasive FNA approach $(6,7)$. The results of ultrasound examination usually determine whether patients should undergo puncture or surgery. The American College of Radiology (ACR) released the thyroid imaging-reporting and data system (TI-RADS) in 2009 to standardize ultrasound results. This specification makes the assessment of benign and malignant thyroid nodules clearer. Application of the TI-RADS classification helps to regulate domestic ultrasound diagnosis; the standard was revised in $2015(8,9)$.

In this paper, the latest medical information technology is used to analyze patient data obtained from Nanjing Medical University in 2017. The value of the TI-RADS for the diagnosis of benign and malignant thyroid nodules is analyzed according to the TI-RADS classification and pathological results. The TI-RADS result suggest whether patients with thyroid nodules should undergo further invasive examinations and provide a reference for diagnosis and treatment.

\section{Methods}

\section{General information}

Clinical data from patients with thyroid nodules was collected from the clinical data center of the First Affiliated Hospital of Nanjing Medical University. This data included basic patient information, clinical diagnoses, laboratory test reports, examination reports, and medical records, etc. Pathological reports are used as the gold standard to classify benign and malignant nodules. Patients with thyroid nodules were selected with the following conditions: (I) patients with benign thyroid nodules: diagnosed as "thyroid nodule" (E04.101), "thyroid tumor" (D44.001), or "thyroid cyst" (E04.102) by a specialist in previous clinical diagnostic records, with a record of thyroid ultrasound, confirmed by pathological analysis as a benign nodule, who were excluded from the diagnosis and treatment records diagnosed by a specialist as a malignant tumor patient; or (II) patients with malignant thyroid nodules: patients diagnosed as "thyroid nodules" (E04.101), "thyroid tumors" (D44.001), "thyroid cyst" (E04.102) or "thyroid malignancy" (C73.x00) by a specialist in previous diagnostic records, with a record of thyroid ultrasound, confirmed by pathological diagnosis as a malignant nodule. Taking the pathological diagnosis date from January $1^{\text {st }}, 2017$ to December $31^{\text {st }}, 2017$ as the query condition, a total of 1,196 pathological reports met the above conditions; then 1,558 ultrasound report and ultrasound images were retrieved from selected patients. Then excluding patients who have not taken thyroid function test in the hospital, excluding patients whose ultrasound examination after the pathological examination, excluding patients without ultrasound examination before thyroidectomy, finally who achieved a one-toone correspondence between their preoperative thyroid function test, preoperative ultrasound and pathological report entered the study cohort. A total of 811 patients were enrolled.

\section{Ultrasound and pathological report analysis}

Radiologists reported the ultrasound records in accordance with the international TI-RADS classification. When the grading judgment of the patient was difficult to define, it was marked as level between the two levels in the report (e.g., 
in patients between $4 \mathrm{C}$ and 5 , the level of the ultrasound report was set as $4 c-5)$. Using natural language processing technology to achieve automatic extraction of nodule position and TI-RADS classification.

The pathologist observed the histological features of the left and right nodules and indicates whether the nodules were benign or malignant in pathological reports. The common pathological diagnoses were papillary thyroid carcinoma, nodular goiter, thyroid follicle gonadal adenomas, and thyroiditis. We classified papillary carcinoma as malignant, while others are benign. Similarly, natural language processing techniques were used to automatically extract nodule sites and to determine the benign and malignant classifications.

\section{Statistical methods}

SPSS 22.0 and the R language platform, RGui (64-bit), were used to perform statistical analyses. A $t$-test was used to analyze the study variables of thyroid nodules. The relationship between patients' basic characteristics and benign and malignant thyroid tumors was analyzed. A $P$ value of $<0.05$ was considered statistically significant. Logistic multiple regression analysis was used to analyze the risk factors for thyroid function in thyroid nodules. We used the TI-RADS classification of ultrasound diagnosis to analyze the effect of classification on the diagnosis of thyroid nodules. Parameters such as sensitivity, specificity, Youden's index, positive predictive value, and negative predictive value were calculated. The receiver operating characteristic (ROC) curve area was calculated to observe the relationship between ultrasound results and the final benign and malignant diagnosis.

\section{Results}

Patients with thyroid nodules were screened using data from the clinical data center of the First Affiliated Hospital of Nanjing Medical University, and information on the preoperative thyroid function test, preoperative ultrasound and pathological report data was collected in 2017. The pathological report results were used as the gold standard for diagnosis of benign and malignant tumors. A total of 811 patients were enrolled. The analysis of basic characteristics is shown in Table 1. There were a total of 161 patients in the benign group and 650 in the malignant group. The data does not indicate the proportion of patients with benign and malignant nodules in the general population. The reason for this proportion is the pathological examination result is the grouping standard while patients who perform pathological examination are often malignant. The result showed that the mean age of patients in the malignant tumor group was lower than the mean age of patients in the benign tumor group. The incidence of malignant nodules in the non-elderly group was significantly greater than that observed in the elderly group $(\mathrm{P}<0.001)$. The ratio of males to females with nodules was 1:3.33. The nodules occurred mostly in middle-aged patients aged 40-60 years, which, accounted for approximately $50 \%$ of nodules. The results were highly consistent with the results of published literature, but gender had no significant effect on the occurrence of malignant nodules $(\mathrm{P}>0.05)$. The incidence of malignant nodules in patients who were married was significantly greater compared with patients who were not married $(\mathrm{P}<0.05)$, while medical insurance status had no significant effect on the incidence of malignant nodules $(\mathrm{P}>0.05)$.

\section{Analysis of correlation nodules classification and thyroid function test results}

The influence of thyroid function index on benign and malignant thyroid nodules was analyzed previously; however, different researchers have different opinions. This paper retrospectively analyzed the influence of thyroid function indicators such as free thyroxine (FT4), free triiodothyronine (FT3), thyroid-stimulating hormone (TSH) and thyroglobulin (TG), on the occurrence of malignant nodules based on 811 patients at the First Affiliated Hospital of Nanjing Medical University. Logistic regression was used to analyze the factors affecting pathology results by univariate and multivariate analyses. $\mathrm{P}<0.01$ was considered statistically significant. Table 2 shows the results of the univariate analysis and the variables that impacted the diagnosis. TG, FT4 and FT3 were key factors in judging whether nodules were benign or malignant. Multivariate logistic regression analysis was used to perform on the variables with significant factors in the univariate analysis, and the independent risk factors and the odds ratios (OR ) value of each factor were calculated. The diagnostic score table was obtained according to the OR values. The ROC curve was drawn and the cutoff value was determined. We constructed a multivariable logistic regression model to predictive thyroid malignancy that includes age, TG, FT4 and FT3 as predictors, which reached an optimism-corrected area under the ROC curve 
Table 1 General characteristics of patients with thyroid nodules

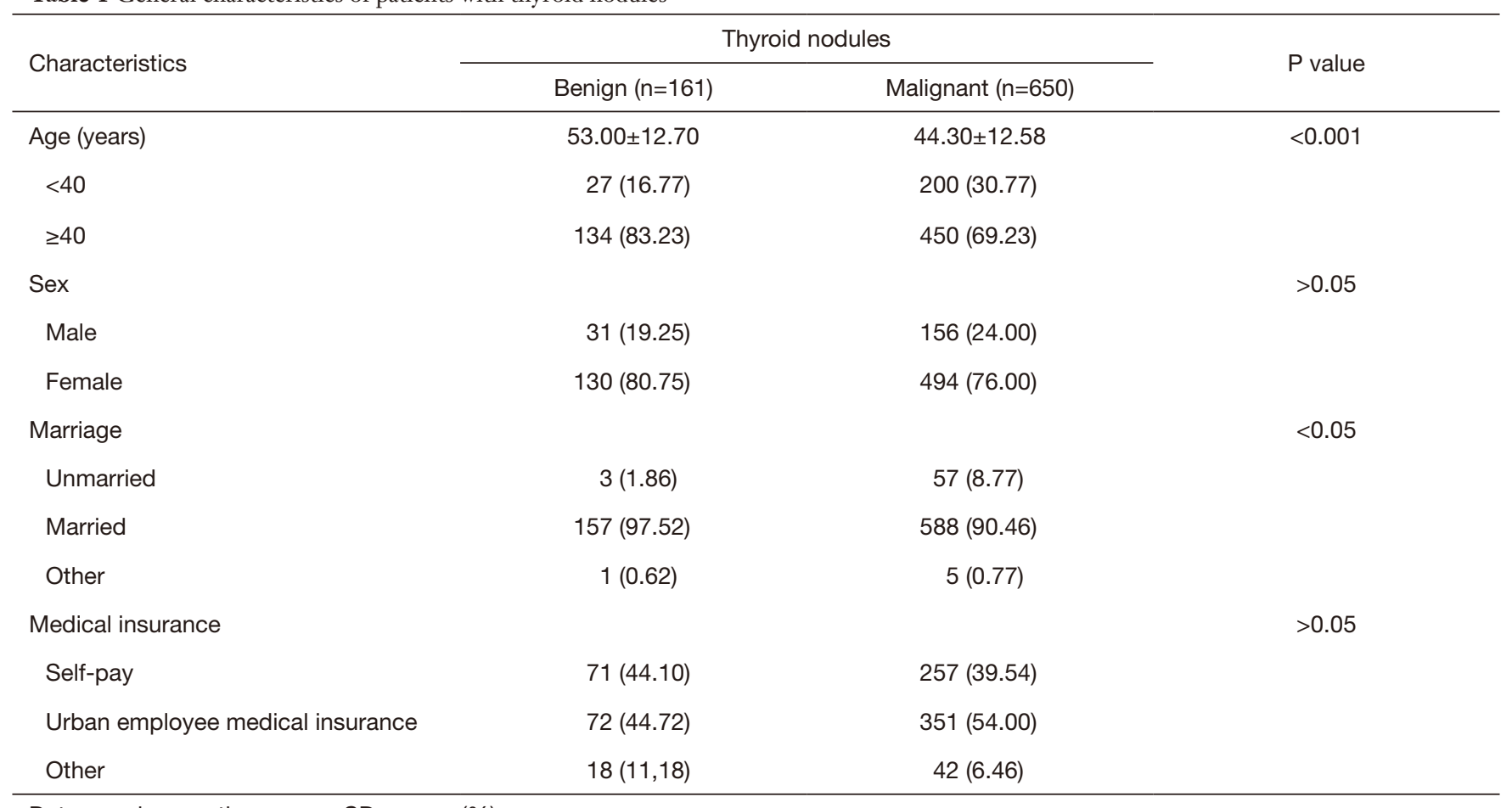

Data are given as the mean \pm SD or as $\mathrm{n}(\%)$.

Table 2 Analysis of the correlation between thyroid function test and nodule classification

\begin{tabular}{|c|c|c|c|c|}
\hline Factor & OR value & $P$ value & \multicolumn{2}{|c|}{ 95\% $\operatorname{EXP}(\mathrm{B})$ confidence interval } \\
\hline Medical insurance & 0.965 & 0.750 & 0.775 & 1.202 \\
\hline Sex & 0.677 & 0.101 & 0.424 & 1.079 \\
\hline Marriage & 0.851 & 0.552 & 0.501 & 1.446 \\
\hline Age & 0.950 & 0.000 & 0.936 & 0.964 \\
\hline TSH & 1.009 & 0.766 & 0.951 & 1.071 \\
\hline TG & 0.996 & 0.000 & 0.994 & 0.998 \\
\hline FT4 & 1.112 & 0.003 & 1.036 & 1.193 \\
\hline
\end{tabular}

TSH, thyroid-stimulating hormone; TG, Thyroglobulin; FT4, free thyroxine; FT3, free triiodothyronine. 


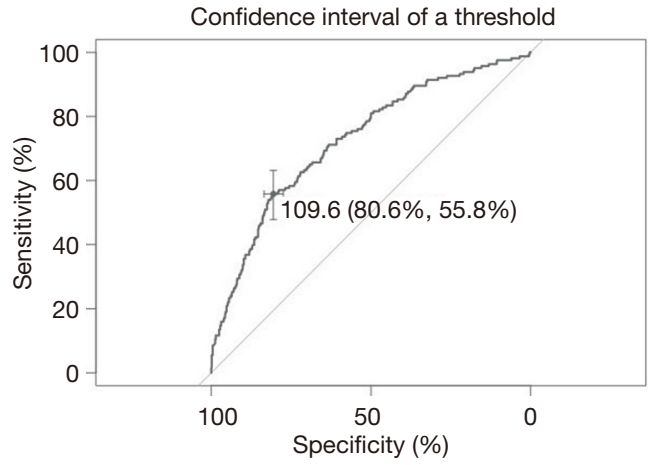

Figure 1 ROC graph based on multivariate analysis. ROC, receiver operating characteristic.

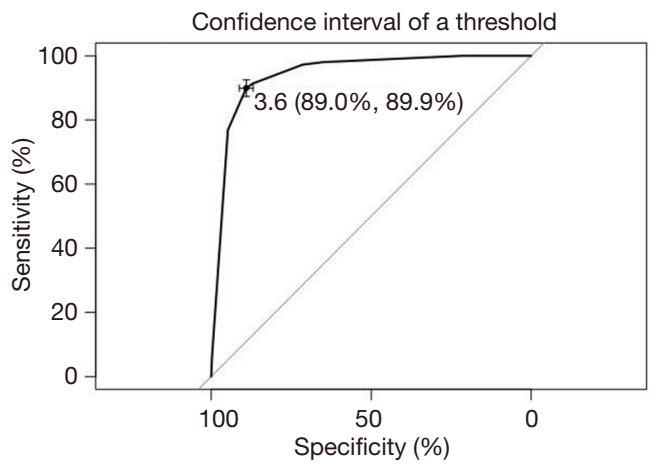

Figure 2 ROC graph based on ultrasound report. ROC, receiver operating characteristic.

(AUC) of 0.727. As shown in the ROC curve in Figure 1, the optimal cutoff level of diagnostic score for predicting the thyroid malignancy was 109.6 , whose sensitivity and specificity were $80.6 \%$ and $55.8 \%$, respectively. The evaluation of predicting the diagnostic accuracy of thyroid malignancies was relatively low.

\section{Comparison between ultrasound reports and pathological reports}

The TI-RADS grading system published by the ACR, provides a relatively objective scoring system for sonographers and surgeons to evaluate in five major areas: nodule composition, echo, morphology, margin, and strong echo. The scoring system of ACR divides the thyroid nodules into five levels. Ultrasound enables clinical experts to diagnose the benign and malignant nodules. In the present study, we diagnosed the benign and malignant nodules using the TI-RADS grading results in ultrasound reports, and evaluated the classification using the accuracy of classification of benign and malignant nodules. In the ultrasound reports, the doctors released inspection reports according to the ACR TI-RADS standards and further refined the grades. The grading was divided into nine levels: 2, 3, 4a, 4a-4b, 4b, 4b-4c, 4c, 4c-5, and 5. If the doctor's grading judgment was difficult to define, the levels intervening between the two levels in the report were marked (e.g., for patients between $4 c$ and 5 , the level of the ultrasound report was $4 \mathrm{c}-5$ ).

Since the results of ultrasonography and pathological diagnosis of the left and right sides were often inconsistent, a total of 1,290 nodules with preoperative ultrasound results and pathological findings from 811 patients were examined. The 1,290 nodules comprised 506 benign nodules and 784 malignant nodules according to the results of pathological diagnoses. For ultrasound grades 2, 3, 4a, 4a-4b, 4b, 4b$4 c, 4 c, 4 c-5$, and 5 , the rate of malignant nodules was $6.06 \%, 9.82 \%, 40.54 \%, 68.18 \%$, 80.39\%, 92.59\%, 97.14\%, $100 \%$, and $100 \%$. As the grade increases, the percentage of malignancy increased in turn. The ROC curve was constructed as shown in Figure 2. ROC analysis indicated the potential predictive value of preoperative ultrasound results with an AUC of 0.942 [95\% confidence interval (CI): 0.930-0.955]. A cut-off of 3.6 (ultrasound grade) was individuated for using preoperative ultrasound results to predict malignant nodules, showing a sensitivity of $89.0 \%$ and a specificity of $89.9 \%$. The sensitivity and specificity of the respective categories of diagnosis are shown in Table 3. The actual malignant percentage has a high degree of compliance with the theoretical malignant percentage, which shows that ultrasonography has good diagnostic value for thyroid nodular disease.

The rate of thyroid nodule malignancy increases continuously with an increase in ultrasound classification from category 2 to category 5 , reaching the highest point on Youden's index at the 4a classification. Using the settings: $4 \mathrm{a}$ as $3.5,4 \mathrm{a}-\mathrm{b}$ as $3.75,4 \mathrm{~b}$ as $4,4 \mathrm{~b}-\mathrm{c}$ as $4.25,4 \mathrm{c}$ as 4.5 to calculate the ROC curve, the best prediction point was grade $4 \mathrm{a}$ which is obviously higher than other categories. Category $4 \mathrm{a}$ achieved a positive predictive value of $93.2 \%$ and a negative predictive value of $84.1 \%$ and its recognition ability to judge whether the nodule was benign or malignant was the highest. The sensitivity of the ROC curve in Figure 2 is $89.0 \%$ and the specificity is $89.9 \%$; significantly greater than the corresponding values in Figure 1 (sensitivity $=80.6 \%$, and the specificity $=55.8 \%$ ). The TI-RADS model requires a grade 4 scoring standard for moderately 
Table 3 TI-RADS diagnostic efficiency (\%)

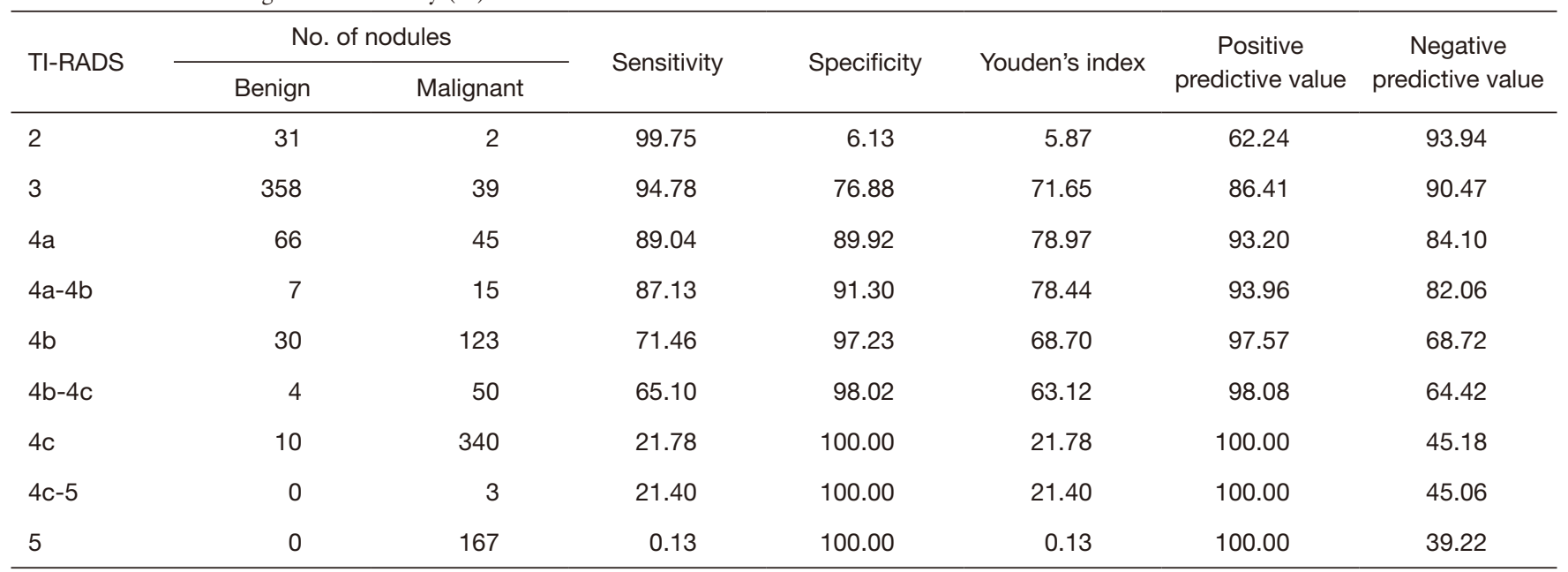

TI-RADS, thyroid imaging-reporting and data system.

suspected malignant risk. The data also demonstrates that the use of the TI-RADS score as a reference for benign and malignant nodules has a high reference value.

\section{Discussion}

\section{Thyroid cancer in young patients}

The ratio of male to female patients with nodules was 1:3.33 and nodules were observed most frequently in middleaged patients aged 40-60 years (approximately 50\%). The average age of patients with malignant nodules was lower. The incidence of nodules in the non-elderly group was significantly greater than the incidence of malignant nodules in the elderly group. One reason for this phenomenon is that patients' senses of health management have increased, and nodules can be detected earlier than before (10). Another reason is that young people's hormone levels are unstable, and life pressures are high, which affects thyroid function, and can induce abnormalities and malignant changes. In particular, hormone levels in young women (e.g., high estrogen progestin ratios owing to the absence of ovulation, and high levels of estrogen during prenatal life reveal the incidence of thyroid nodules (11).

\section{Association between thyroid function tests and nodule classification}

Thyroid cancer and thyroid nodules are the most prevalent form of thyroid endocrine disorder. In our study, we analyzed the classification of benign and malignant nodules using measures of thyroid function. Changes in FT4, FT3, TSH, and TG are common indicators of changes in thyroid function (12-15). These indicators were analyzed and modeled; both FT4 and FT3 had significant effects on malignant nodules and were key factors that could be used to diagnose benign and malignant nodules. The results are consistent with those of a previously published study (16). The risk of papillary thyroid cancer was also inversely associated with serum FT3 in all participants, and was positively associated with the serum FT4. The present study showed only a minimal association with TSH, suggesting that the hypothalamic-pituitary axis is less affected by thyroid nodules. TG is also closely related to benign and malignant outcomes.

\section{Advantages of ultrasound}

Ultrasound image features are key in the diagnosis of benign and malignant thyroid nodules (17-20). In this paper, the TI-RADS and patients' thyroid functions were analyzed separately. The comparative analysis found that ultrasound diagnosis has obvious advantages for the analysis of benign and malignant nodules. The percentage of malignant nodules according to the ultrasound classification increased from grade 2 to grade 5 . Since the diagnosis level of each radiologist is different, methods to reduce the rate of misdiagnosis and improve diagnostic accuracy should be identified (21-23). Particularly for reports with scores of 4 or greater, the results have a significant impact on the 
patients' further treatment options. This study showed that the ultrasound classification $4 \mathrm{a}$ achieved the best benign and malignant classification with a positive predictive value of $93.2 \%$ and a negative predictive value of $84.1 \%$. The study also verified the effectiveness of the TI-RADS classification for the diagnosis of benign and malignant thyroid nodules.

\section{Conclusions}

At present, imaging-based diagnosis and medical care explore in cancer analysis and diagnosis. The present study compared ultrasound reports with thyroid function for nodule classification. The study verifies use of the ACR TIRADS classification for the diagnosis of thyroid nodules, whether benign or malignant. Subsequent research should further analyze ultrasound images, extract key image features, and model them together with existing clinical variables, using a convolutional neural network to introduce an unbiased computer-aided diagnostic tool to assist radiologist with less experience. Further studies should optimize diagnosis using artificial intelligence and provide the clinic with more accurate information to assist clinical decision making.

\section{Acknowledgments}

Funding: The research was supported by grants from the National Key Research \& Development Plan of Ministry of Science and Technology of China (Grant No. 2018YFC1314900), the 2019 Natural Science Research Projects of Universities in Jiangsu Province (19KJB310009).

\section{Footnote}

Conflicts of Interest: All authors have completed the ICMJE uniform disclosure form (available at http://dx.doi. org/10.21037/gs.2020.04.03). The authors have no conflicts of interest to declare.

Ethical Statement: The authors are accountable for all aspects of the work in ensuring that questions related to the accuracy or integrity of any part of the work are appropriately investigated and resolved. The study was approved by the Ethics Committee of the First Affiliated Hospital of Nanjing Medical University (2016-SR-242). As this study is a retrospective study and the contents of the study do not involve personal privacy, the ethics committee approves this study is exempt from the informed consent of participants.

Open Access Statement: This is an Open Access article distributed in accordance with the Creative Commons Attribution-NonCommercial-NoDerivs 4.0 International License (CC BY-NC-ND 4.0), which permits the noncommercial replication and distribution of the article with the strict proviso that no changes or edits are made and the original work is properly cited (including links to both the formal publication through the relevant DOI and the license). See: https://creativecommons.org/licenses/by-nc-nd/4.0/.

\section{References}

1. Pellegriti G, Frasca F, Regalbuto C, et al. Worldwide Increasing Incidence of Thyroid Cancer: Update on Epidemiology and Risk Factors. J Cancer Epidemiol 2013;2013:965212.

2. Ullmann TM, Gray KD, Moore MD, et al. Current controversies and future directions in the diagnosis and management of differentiated thyroid cancers. Gland Surg 2018;7:473-86.

3. Lin JD, Chao TC, Huang BY, et al. Thyroid cancer in the thyroid nodules evaluated by ultrasonography and fineneedle aspiration cytology. Thyroid 2005;15:708-17.

4. Nikiforov YE, Seethala RR, Tallini G, et al. Nomenclature revision for encapsulated follicular variant of papillary thyroid carcinoma: a paradigm shift to reduce overtreatment of indolent tumors. JAMA Oncol 2016;2:1023-9.

5. Nix P, Nicolaides A, Coatesworth AP. Thyroid cancer review 1: presentation and investigation of thyroid cancer. Int J Clin Pract 2005;59:1340-4.

6. Zhang B, Tian J, Pei SF, et al. Machine LearningAssisted System for Thyroid Nodule Diagnosis. Thyroid 2019,29:858-67.

7. Grani G, Lamartina L, Ascoli V, et al. Ultrasonography scoring systems can rule out malignancy in cytologically indeterminate thyroid nodules. Endocrine 2017;57:256-61.

8. Haugen BR, Alexander EK, Bible KC, et al. 2015 American Thyroid Association Management Guidelines for Adult Patients with Thyroid Nodules and Differentiated Thyroid Cancer: The American Thyroid Association Guidelines Task Force on Thyroid Nodules and Differentiated Thyroid Cancer. Thyroid 2016;26:1-133.

9. Rosario PW, Alexandre LS, Nunes MB, et al. Risk of 
Malignancy in Thyroid Nodules Using the American

College of Radiology Thyroid Imaging Reporting and Data System in the NIFTP Era. Horm Metab Res 2018;50:735-7.

10. Araque DVP, Bleyer A, Brito JP, et al. Thyroid cancer in adolescents and young adults. Future Oncol 2017;13:1253-61.

11. Kowalczyk K, Franik G, Kowalczyk D, et al. Thyroid disorders in polycystic ovary syndrome. Eur Rev Med Pharmacol Sci 2017;21:346-60.

12. Pacini F. Thyroid function: optimizing molecular testing in thyroid nodule cytology. Nat Rev Endocrinol 2012;8:390-1.

13. Partyka KL, Trevino K, Randolph ML, et al. Risk of malignancy and neoplasia predicted by three molecular testing platforms in indeterminate thyroid nodules on fineneedle aspiration. Diagn Cytopathol 2019;47:853-62.

14. Ollero MD, Toni M, Pineda JJ, et al. Thyroid function reference values in healthy iodine-sufficient pregnant women and influence of thyroid nodules on thyroidstimulating hormone and free thyroxine values. Thyroid 2019;29:421-9.

15. Su JJ, Hui LZ, Xi CJ, et al. Correlation analysis of ultrasonic characteristics, pathological type, and molecular markers of thyroid nodules. Genet Mol Res 2015;14:9-20.

16. Hu MJ, Zhang C, Liang L, et al. Fasting serum glucose, thyroid-stimulating hormone, and thyroid hormones and

Cite this article as: Miao S, Jing M, Sheng R, Cui D, Lu S, Zhang X, Jing S, Zhang X, Shan T, Shan H, Xu T, Wang B, Wang Z, Liu Y. The analysis of differential diagnosis of benign and malignant thyroid nodules based on ultrasound reports. Gland Surg 2020;9(3):653-660. doi: 10.21037/gs.2020.04.03 risk of papillary thyroid cancer: A case-control study. Head Neck 2019;41:2277-84.

17. Popli MB, Rastogi A, Bhalla P, et al. Utility of gray-scale ultrasound to differentiate benign from malignant thyroid nodules. Indian J Radiol Imaging 2012;22:63-8.

18. Brito JP, Gionfriddo MR, Nofal AA, et al. The Accuracy of Thyroid Nodule Ultrasound to Predict Thyroid Cancer: Systematic Review and Meta-Analysis. J Clin Endocrinol Metab 2014;99:1253-63.

19. Haymart MR, Banerjee M, Reyes-Gastelum D, et al. Thyroid Ultrasound and the Increase in Diagnosis of Low-Risk Thyroid Cancer. J Clin Endocrinol Metab 2019;104:785-92.

20. Rocha TG, Rosario PW, Silva AL, et al. Ultrasonography Classification of the American Thyroid Association for Predicting Malignancy in Thyroid Nodules $>1 \mathrm{~cm}$ with Indeterminate Cytology: A Prospective Study. Horm Metab Res 2018;50:597-601.

21. Gamme G, Parrington T, Wiebe E, et al. The utility of thyroid ultrasonography in the management of thyroid nodules. Can J Surg 2017;60:134-9.

22. Baig FN, Lunenburg JTJV, Liu SYW, et al. Computeraided assessment of regional vascularity of thyroid nodules for prediction of malignancy. Sci Rep 2017;7:14350.

23. Keramidas EG, Maroulis D, Lakovidis DK, et al. TND: a thyroid nodule detection system for analysis of ultrasound images and videos. J Med Syst 2012;36:1271-81. 